\title{
Standards from Birth to Maturity for Height, Weight, Height Velocity, and Weight Velocity: British Children, I965
}

\author{
Part $I^{\star}$ \\ J. M. TANNER, R. H. WHITEHOUSE, and M. TAKAISHI $\dagger$ \\ From the Department of Growth and Development, Institute of Child Health, University of London, \\ Guilford Street, London, W.C.1
}

The accurate assessment of the physical growth and development of children has recently come to interest paediatricians and public health officers much more than hitherto. In the developed countries, where infectious diseases are well controlled, an increasing proportion of children referred to paediatricians present a disorder of growth either as a primary symptom or associated with some metabolic defect; and in underdeveloped countries children's growth is now widely recognized as a sensitive index of the health and nutrition of the population. This paper seeks to clarify some aspects of the assessment of growth, particularly in clinical paediatrics, and gives, for the first time, standards for height and weight velocity from birth to maturity, as well as for height and weight attained at each year of age.

The principal questions that the physician has commonly to answer concerning a child's growth are:

(1) Is this child's size within normal limits for his age, sex, population, and socio-economic group ?

(2) Has this child's rate of growth over the past year, say, been within normal limits for his age, sex etc?

(3) Has my treatment produced a change in the rate of growth of this child?

The first question arises when the child is seen for the first time in the clinic, in school, or perhaps in a public health screening survey. It requires standards of size attained at each age, which we shall refer to as 'distance' standards, on the analogy of a child having travelled a certain distance along the way to

\footnotetext{
Received February 21, 1966.

* Part II of this article will appear in the next issue, and will contain the references for the complete article, the acknowledgements, and the appendices.

t On study leave from the Institute of Public Health, Tokyo, Japan.
}

adult size. Charts of height and weight attained at each age exist for children of many countries; a typical example is our own for British children (Tanner, 1958; Tanner and Whitehouse, 1959)* based for the most part on London boys and girls measured in 1954, supplemented with Oxford children measured in the period 1946-54. But children in many countries have been getting larger during the past 50 years or more, both because they are growing up faster and so reaching adult size at an earlier age, and because their adult size itself is increasing (Tanner, 1962a). Hence the height-forage and weight-for-age standards have to be revised every 10 or 15 years until this trend stops. We therefore present here new standards based mostly on London children measured in 1959 and adjusted slightly so as to be appropriate for 1965 . These standards are adequate for judging the normality or otherwise of a child when is he seen on one occasion only. But they need supplementing if the child has been seen on several occasions, i.e. if two or more plots on his growth chart are being considered (see question 4, below).

The second, more searching, question requires standards for velocity or rate of growth. These have not so far been available over the whole age range. Height velocity curves from birth to maturity for the typical, or 50th centile, boy and girl were published in an article of which this paper is a further development (Tanner, 1962b), and have been used in clinical problems of growth regulation (Prader, Tanner, and von Harnack, 1963). We give here the other standard lines as well as the 50th.

The third question really requires standards for

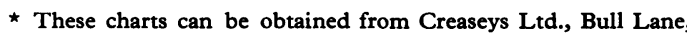
Hertford. Two sizes are obtainable, the smaller designed to fit in hospital note folders and the larger double foolscap size for more accurate plotting. 
acceleration or rate of change of growth velocity. It is not possible at present to give such standards. But just as, in the past, abnormality of velocity could be detected, though not quantified, from distance plots, so unusual accelerations can be detected from the new velocity charts given here. Though children do not tend to stay at a given velocity centile during growth nearly so closely as at a given distance centile, a marked change of velocity centile does still signal an important acceleration or deceleration. Such accelerations are much less easily seen in distance charts.

There is still a fourth question, which is biologically the most fundamental, and is a variant and extension of question 2 . It is: (4) To what extent has this child's growth conformed to the normal pattern over the whole of the last so many, say 6 , or 10 years? Clearly this question also underlies question 3 when it concerns the effect of a long-term disease or a long-term treatment. This fourth question poses formidable methodological difficulties, and we cannot provide a full and precise answer to it at present.

The clinician has long been accustomed to judging this roughly by comparing the curve of a child's growth with the centile curves plotted on the conventional distance charts. He does this because most children stay approximately at the same centile (or in the same channel, as it is sometimes called) during most of their growth, and on average they return to this centile when growth is completed. At adolescence, however, this technique for assessing growth fails, because at this time the healthy child departs from the centile he has hitherto been following. If he is an early maturer he moves to a higher c centile before, usually, dropping back at maturity to his pre-adolescent position; if he matures late, he moves to a lower centile and then catches up later to his previous position. This occurs because some children have their adolescent growth spurts early and some late and these 'phase differences' produce an alteration in the shape of the conventional centiles in a manner discussed more fully below. Thus at adolescence the conventional height-attained and weight-attained charts are definitely misleading if they are used to judge a child's growth progress. To restore the usual use of the standards in judging progress, therefore, we have put in on the charts a system of auxiliary curves which $d o$ have the shape of an individual's plot of growth at this time. This does at least remove one of the many difficulties involved in answering question 4.

The measurements of height and weight alone, of course, by no means represent a full assessment of the child's physical development. In public health surveys the amount of subcutaneous fat may be the next most important item, and standards for skinfold measurements at two sites of the body are now available (Tanner and Whitehouse, 1962). In clinical work, particularly endocrinology, measurements of the relation of limb and trunk length are considered important and standards for this are in preparation. At the Growth Disorder Clinic of The Hospital for Sick Children we also take measurements of shoulder and hip widths, elbow and knee widths, and arm, calf, and thigh circumferences. An assessment of skeletal maturity by wrist and hand $X$ ray is an essential part of the whole examination (Tanner, Whitehouse, and Healy, 1962); and an assessment of dental maturity is also often helpful.

This paper deals only with height and weight. There follows (A) a description of the data on which the standards are based; (B) new cross-sectional-type standards for height and weight attained, together with an account of our development of these, so that they give additional information regarding the course of growth of a single individual followed longitudinally; (C) velocity standards for height and weight with an account of the method of their construction; and (D) an example of the use of both standards in clinical practice.

Portions of this paper are technical, and though we hope they are interesting to other auxologists and to potential critics they can be skipped without detriment to the main argument or to the understanding of the standards. We have placed these portions in small print. Paediatricians who are simply concerned with using the standards, therefore, are advised to skip the small-print sections (which constitute $40 \%$ of the paper).

\section{(A) Data on Which Standards are Based}

(a) Distance standards. Supine lengths, heights, and weights from birth to $5 \frac{1}{2}$ years are based on the sample of about 80 children of each sex followed longitudinally at the Child Study Centre in London (now the Centre for the Study of Human Development; see Moore, Hindley, and Falkner, 1954). The measurement of height was done as described below; supine length was taken with the child stretched maximally on a special table, fitted with a sliding footboard with a recording counter operated by a rack-and-pinion. The mother or nurse held the child's head in contact with the headboard at the top of the table, and the measurer then stretched the child, straightened the legs, turned the feet toes upwards and brought the sliding board into contact with the child's heels. This sample was drawn at random from families living in a central area of London, but is very small indeed for serving as a standard. However, the Child Study Centre data agree closely with data from the Oxford Child Health Survey, on 250 children of each sex at each age (see Tanner, 1958) and, in respect of velocity, 
with the Swiss data of Heierli (1960) and the Edinburgh data of Thomson (1956a, b).

From $5 \frac{1}{2}$ to $15 \frac{1}{2}$ the source of our normal data was the London County Council survey of 1959 (Scott, 1961). In this survey a random sample of London schools was taken, of a number producing approximately 1,000 boys and 1,000 girls at each year of age. All children in the selected schools were measured. Nine pairs of measurers and recorders were specially trained by us in measuring techniques, and all measurements of heights and weights were taken by one of these pairs. Height was taken with the child stretched up maximally, gentle traction being applied under the mastoid processes by the measurer. The child stood against the wall, on which had been fixed a wooden measuring rod reading in millimetres. A right-angled block was slid down the wall until the bottom surface touched the child's head. The head was kept in the Frankfurt plane and the heels checked to make sure they were on the ground. Weight was measured in under-pants and vest only, with the weighing scale previously adjusted to zero while carrying a sample vest and under-pants; thus nude weight was recorded.

The standard curves are much less accurately determined from $15 \frac{1}{2}$ onwards. The London County Council survey includes some children of each sex at $16 \frac{1}{2}$, but from this age onwards we have had to rely on the increments for some 30 children in the Harpenden Growth Study, who have been studied each year till age 20 . There are no really satisfactory data for the mature height of 20-yearold men and women in Great Britain, but our figure agrees reasonably well with the figures available for Service populations. For example the average height of National Service Army recruits in 1951 (accepted virtually without reference to a minimum) was measured as about $172 \mathrm{~cm}$. at 18 rising to $173 \mathrm{~cm}$. at 20 (Rosenbaum, 1954). As our method of measuring height almost certainly produces higher figures, our mean of $175 \mathrm{~cm}$. seems approximately correct for young men in the early 1960's.

Supine lengths are given from 1 month to 2 years and standing heights thereafter. The break in the standards represents the difference between the two measurements; the average difference is a little under a centimetre, but unfortunately children differ considerably, even at ages well above 2 years (cf. French data in Sempé, Tutin, and Masse, 1964). Thus in passing from one measurement to the other a difference of from zero to $2.5 \mathrm{~cm}$. may occur; supine length is very seldom actually less than height. At ages between 2 and 3 years inclusive, both measurements should be done so that an overlap is recorded and calculation of velocity made possible. The velocity curve is not affected by the change of method of measurement, since supine length increments equal, at least approximately, the corresponding height increments.

(b) Velocity standards. Annual increments were available for children in the Child Study Centre group up to $11-12$, and these were used in the manner described in the section below on construction of velocity standards. During adolescence the basic data consisted of three-monthly measurements of a numter of children in the Harpenden Growth Study followed throughout the whole of adolescence. The nature of this sample is described below, with the manner in which the data were used.

\section{(B) Cross-sectional Type Standards for Height and Weight Attained: 1965}

For the construction of distance standards, the values for height and weight in the data above were plotted against age. For height, a Gaussian distribution was assumed and centiles calculated from the mean and standard deviation at each age, since this is a more precise method of estimating them than calculation from raw data, provided the distribution is normal (see Tanner, 1952). The 50th centile is then the mean, the 3rd and 97th centiles $\pm 1 \cdot 881$ SDs, 10th and 90th $\pm 1 \cdot 282$ SDs, and 25 th and 75 th $\pm 0 \cdot 675$ SDs.

First, however, a correction has to be made at those ages where cross-sectional data are used as the basis for a standard (L.C.C. data ages $5 \frac{1}{2}$ to $15 \frac{1}{2}$ ). The standard deviation has to be reduced from a figure covering the whole year interval such as 5.0 to 5.9 to an instantaneous SD' giving the variation at, say, $5 \cdot 5$ years precisely, since it is against this that an individual child has to be judged. The method for doing this is given by Healy (1962). The distribution of height is not in fact strictly Gaussian at adolescence for reasons first given by Boas in 1892 (see Tanner, 1959, p. 78), but the deviation is not quantitatively very important. For the weights, the distribution of which is considerably skewed, the published L.C.C. sample centiles were used, interpolated directly from the raw data but scaled in towards the median by the ratio of the whole-year SD to the instantaneous SD.

In the Child Study Centre data the centiles for height attained were calculated from the standard deviations without correction, since the children were all measured within a few weeks of their birthday or other fixed age. The standard deviations were plotted, and a smooth curve was drawn through them, boys and girls being averaged up to 8 years, since no consistent sex difference in SD in this age range was visible. The Child Study Centre, Oxford, and L.C.C. data agreed well; in comparison the Swiss data of Heierli (1960) and the French series reported by Sempé et al. (1964) give rather lower SDs from 2 to 4 , perhaps due to restrictions in sampling. The SDs for final mature height were estimated from the same sources as mean mature height (Rosenbaum, 1954; Tanner and Whitehouse, 1959; Joint Clothing Council, 1957). They lie at $6 \cdot 65 \mathrm{~cm}$. for men and $6.0 \mathrm{~cm}$. for women. The weight centiles were estimated directly from the data and the resultant curves smoothed. The figures agreed well with the much larger series of weights collected by the Ministry of Health (1959) from ages 0 to 3 .

Smoothing the centiles was done by eye, but very 
little was necessary. These are now cross-sectional type standards, and as such should be used when assessing any single (e.g. first-time) measurement of a child. The values of the centiles are tabulated in Appendix Tables III and IV. In Fig. 12, 13, 14, and 15, p. 468-471 below, the cross-sectional standards are those without the shading.

\section{'Phase-difference' Effect in Conventional Distance Standards}

The usefulness of conventional distance-attained standards decreases at adolescence because the centiles move further apart as a result of what we term the 'phase-difference' effect. Furthermore, in clinical work or in any research where a child is followed longitudinally, the additional information supplied by successive measurements, i.e. the shape of the child's curve, can be used to make a more critical assessment of the child's status. This can only be done, however, if charts are available with curves that are the correct shape for the individual. This the conventional distance curves are not, because the phase difference affects them in a way we shall now describe.

When adolescence begins the child's growth rate rises sharply; a peak is reached, followed by a sharp decline. This growth spurt has a similar form in all children, but the age of its occurrence varies greatly, just as does the age of menarche, or the age of appearance of the pubic hair. The effect of this age variation on standards derived from measuring one set of children aged 9, another set aged 10 , and so on (cross-section-type standards) is illustrated in Fig. 1. In 1a (upper) the solid lines represent the curves of height velocity of five boys each followed throughout adolescence in the Harpenden Growth Study. The heavy interrupted line represents the mean of these curves, obtained simply by taking the average of the five individual values at each age. This mean curve in no way characterizes any individual; it smoothes out the spurt, spreading it along the age axis. It does not take account of the 'phasedifferences' between the individual curves. Fig. 1b (below) shows the same individual curves, but arranged so that their peak velocities coincide; the average curve then characterizes the group in a proper manner. Boas drew attention to this in 1892, and Davenport, Backman, and Shuttleworth later each made the same point (see Tanner, 1962a, p. 10): but no practical result followed.

Averages computed from cross-sectional or mass survey data, in which each child is only measured at one age, inevitably produce velocity curves of this flattened type, instead of curves representing the progress of an individual. Height-attained, or distance, curves are the integrals of velocity curves, and show this flattening by not rising sufficiently sharply at adolescence. In consequence, as shown in Fig. 2 left, a healthy child does not follow the centile lines at adolescence. If he is an early maturing boy he rises through the centiles and subsequently falls back to regain, on average, his position before puberty (M613). If he is a late-maturing boy he falls through the centiles first and then regains rapidly his earlier status (M112). The boy who has an average peak velocity and attains it at the average chronological age will follow the solid thick line in Fig. 2, right. This represents the 50th centile of the new 'typical-individual' standard plotted on the previous (Tanner and Whitehouse, 1959) British charts.

\section{Determining the Shape of the Individual Curve at Adolescence}

The shape of the curve at adolescence must be determined from longitudinal data, that is from measurements on individuals followed throughout puberty. The individual curves may then be combined to produce the proper average, or typicalindividual, curve. This is most conveniently done by combining the individual velocity rather than distance curves, in the manner of Fig. $1 \mathrm{~b}$, and then integrating the result. In the records of the Harpenden Growth Study we had available measurements of height on 49 healthy boys and 41 healthy girls followed for a sufficient period before, during, and after puberty to give us a clear indication of the course of their adolescent growth spurts. These data have two advantages over most other published longitudinal measurements. First, all measurements were taken by the same measurer throughout; second, measurements were taken at three-monthly intervals during the three or four years of each individual's spurt, and at six-monthly intervals before and after this.

Method of smoothing data. Each individual's empirical data have to be smoothed by some procedure, graphical or computational, to remove measuring error, the effects of seasonal variation, and any minor variations due to other causes. An example of how we did the smoothing is shown in Fig. 3. The individual's height measurements at each age were plotted, and a smooth curve (A) drawn through them, using a flexible lead spline as a guide. The increments of this fitted distance curve were then read off at three-month intervals, converted to $\mathrm{cm}$./yr., and plotted as the solid points in Fig. 3. The velocity curve (solid line B) was then drawn by eye through these points. After this had been done the actual increments given by the individual's measurements were plotted and are indicated by the 


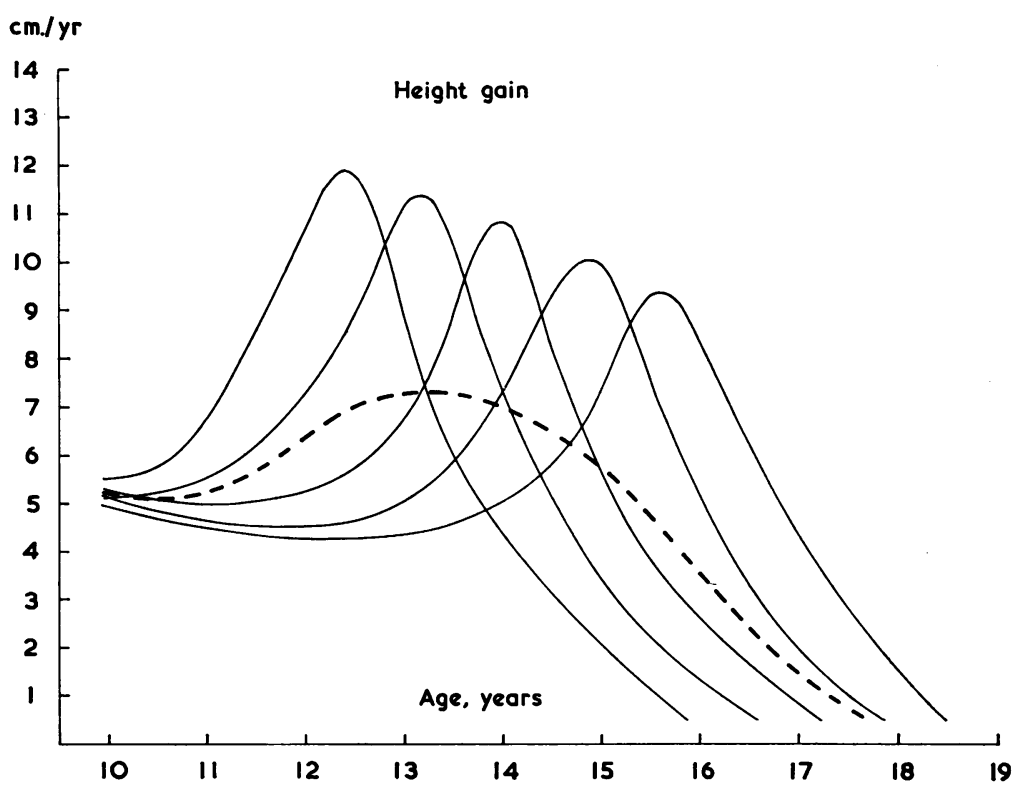

(A)

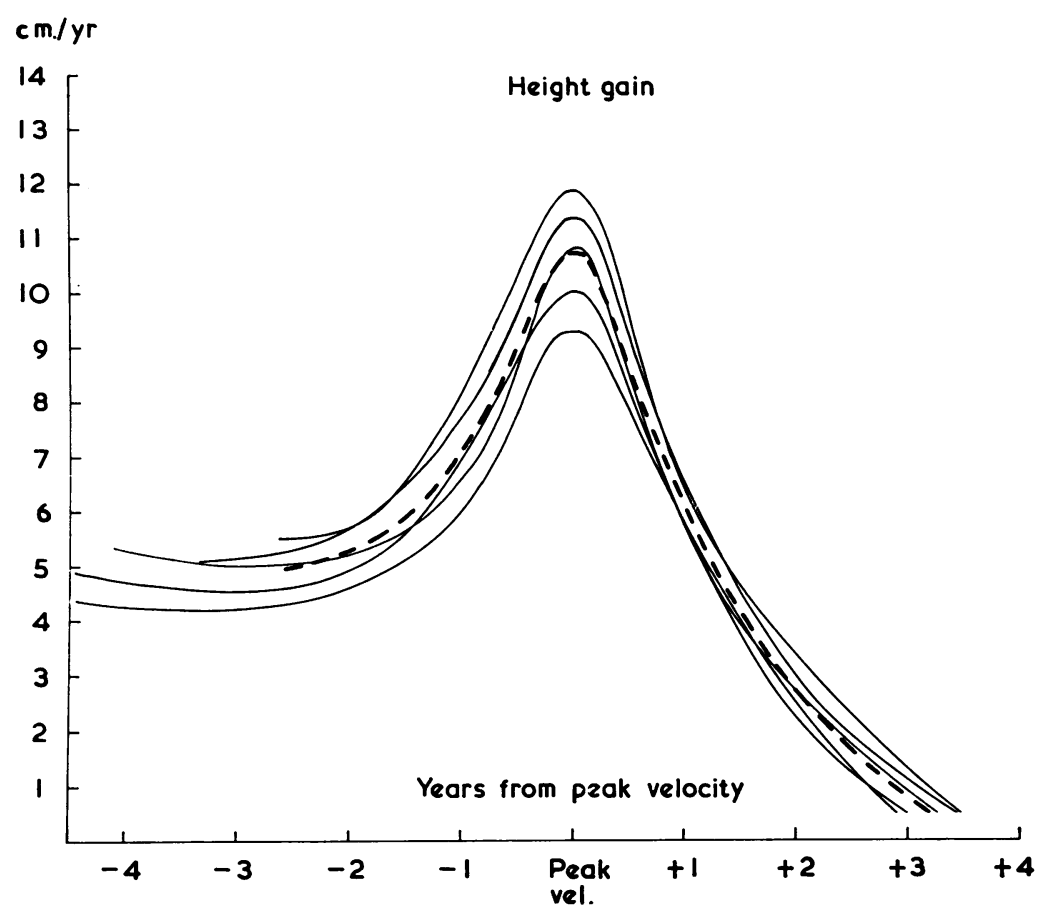

(B)

FIG. 1.-The relation between individual and mean velocities during the adolescent spurt. (A) the individual height velocity curves of 5 boys of the Harpenden Growth Study (solid lines) with the mean curve (dashed) constructed by averaging their values at each age. (B) the same curves all plotted according to their peak height velocity. 

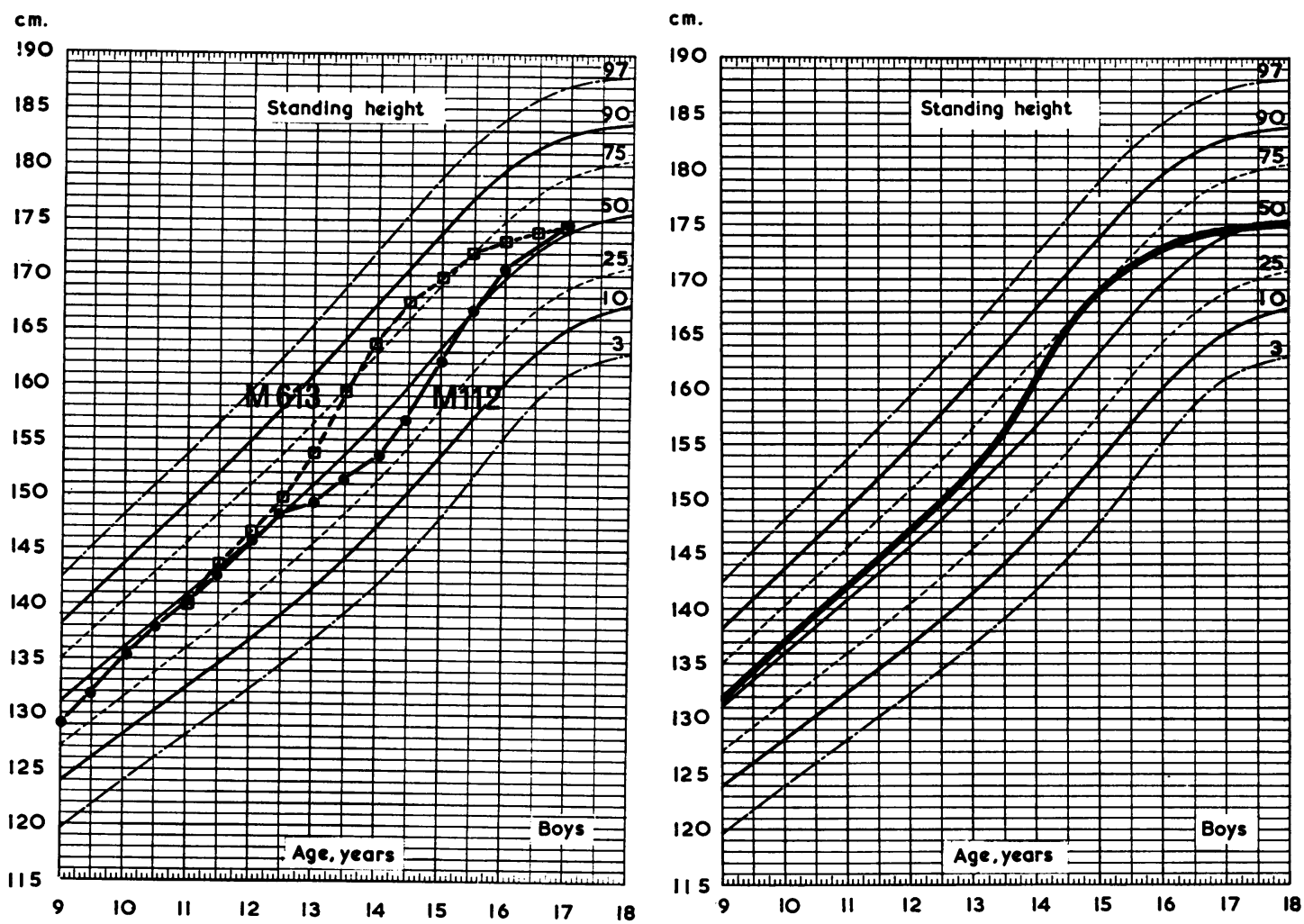

FIG. 2.-Left, height-attained curves of an early-and a late-maturing boy, both of average height before and after puberty, plotted on the previous (1959) British height-for-age standards. Right, the present individual-type 50th centile (thick line) plotted on the 1959 standards.

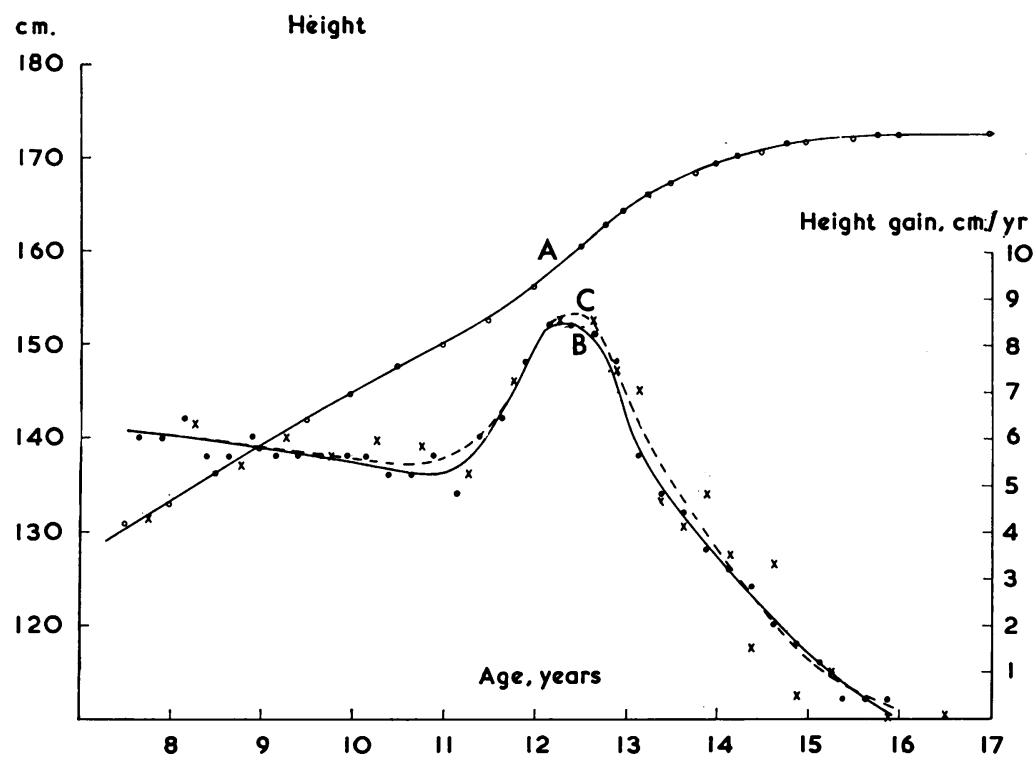

Fig. 3.-Example of graphical fitting procedure. Open circles represent height attained at each age (curve $A$ ); solid dots represent increments derived from the smoothed curve $A$. Solid-line velocity curve $B$ is fitted by eye to the solid dots. Then actual increments are plotted as crosses, and final velocity curve $C$ (dashed line) drawn by modifying curve $B$ in accordance with crosses. 


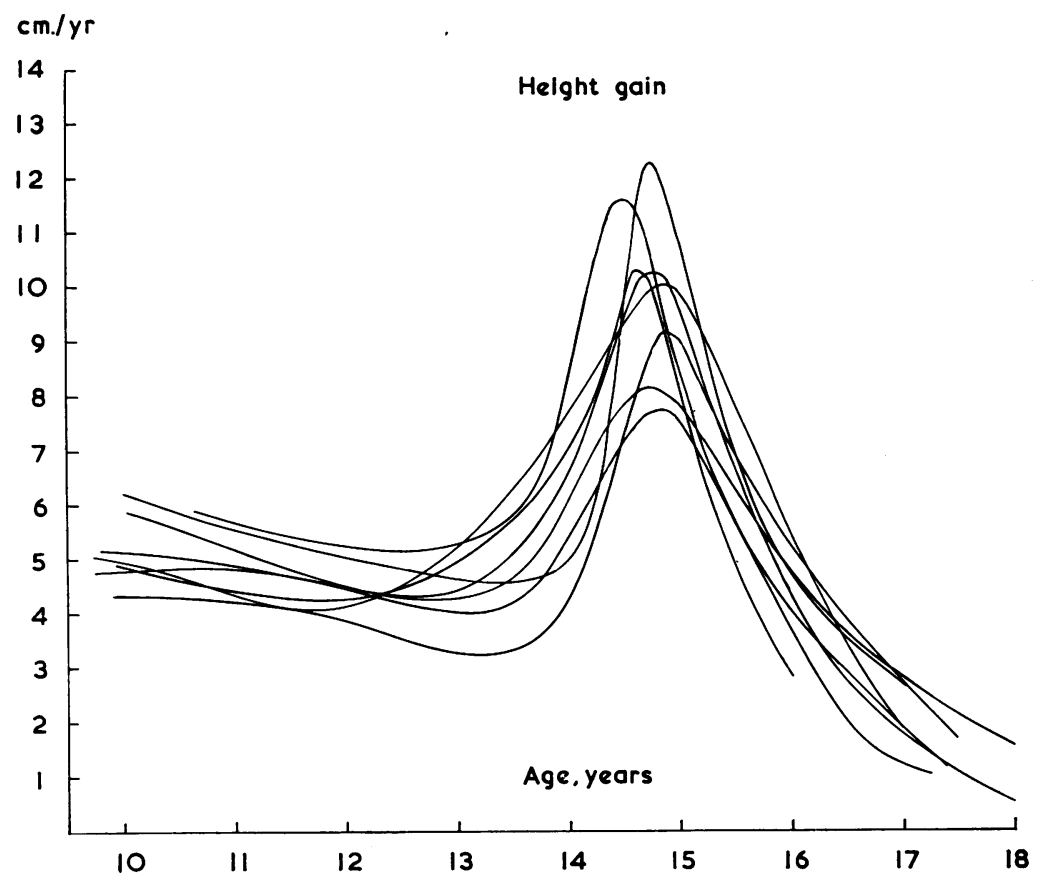

(A)

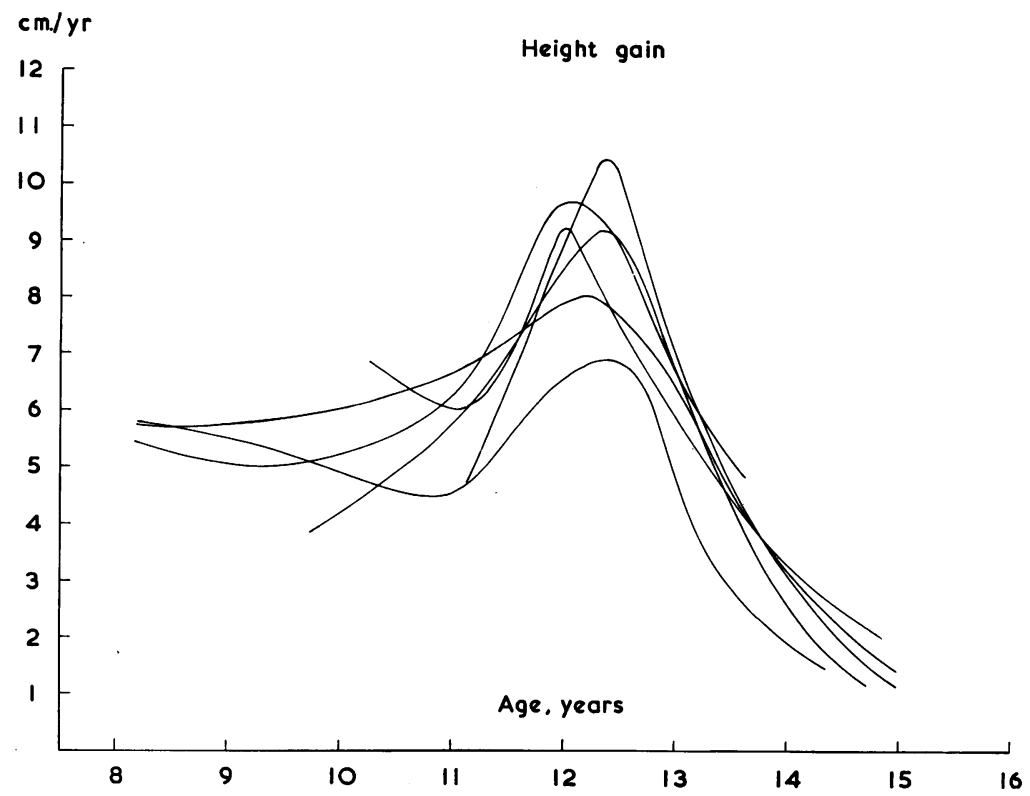

(B)

FIG. 4.-Height velocity curves of individual children. (A) 8 boys with peak height velocity between $14 \cdot 5$ and $14 \cdot 9$; (B) 6 girls with peak height velocity between $12 \cdot 0$ and $12 \cdot 4$. 
crosses. The positions of these crosses were then compared with the velocity curve; if it seemed necessary, the velocity curve was adjusted, again by eye; this is the dashed curve (C). Thus three cycles of smoothing were performed. This is clearly only an approximate method; we have not shown our best-fitting case in Fig. 3, but neither have we exposed the worst. We did not use mathematical methods of fitting because the Gompertz function, which is the curve usually thought to fit the adolescent spurt best, could not be fitted efficiently and expeditiously by a computer program at the time of writing. The method of fit used by Deming (1957) in her otherwise excellent paper on the use of this curve at adolescence is not statistically efficient, and probably produces somewhat biased estimates of the parameters. However, Deming's data, from 24 boys and 24 girls at Denver, mostly measured three-monthly, resemble ours more closely than do any others in the literature. It is encouraging therefore that her results and ours also closely resemble each other.

There is perhaps one advantage in fitting graphically rather than by computer. We were not constrained by having to fit a preselected curve of known form. If we thought it reasonable we could let a dip in the curve remain, rather than smooth it out. In fact, we seldom did this; we were prejudiced in favour of a general unformity of results and of a regularly-shaped velocity curve during the spurt. A selection of our curves is shown in Fig. 4. In $4 \mathrm{~A}$ are the curves for eight boys maturing at near the average age, with peak height velocities between 14.5 and 14.9 years. In $4 B$ are the curves for six early-maturing girls, with peak height velocities between $12 \cdot 0$ and $12 \cdot 4$.

Combining individual curves. Each individual's curve was drawn out on tracing paper, and eight or nine of these, constituting a group such as those illustrated in Fig. 4, were placed one on top of the other and transilluminated from below. The curves were then moved along the time axis until their peaks lay one above the other in a vertical line. An average curve was then drawn over them by eye, as in Fig. 5A. The five average curves for boys resulting from this are shown in Fig. 5B, together with the over-all average curve (dashed), resulting from the combination of these five curves. This was done only to determine the shape of the average curve, not its position in regard to age nor the height of its peak. These were determined more conventionally by reading off the height of each individual's peak and the age at which it occurred and averaging each of these figures over all the individuals. These statistics are shown in Table $I$.

Height. The boy's peak height velocity averages $10.3 \pm 0.22 \mathrm{~cm} . / \mathrm{yr}$. and the girl's $9.0 \pm 0.16 \mathrm{~cm}$. $/ \mathrm{yr}$. The difference between these is very highly significant. Deming's corresponding figures are $9.6 \pm 0.18 \mathrm{~cm}$. $/ \mathrm{yr}$. for boys and $9.1 \pm 0.25 \mathrm{~cm}$. $/ \mathrm{yr}$. for girls. The values for girls agree closely, but our boys' peak is $0.7 \mathrm{~cm} . / \mathrm{yr}$. higher than Deming's. This may just be due to a sampling error, or more likely, to a difference in fitting procedure where it concerns the more highly peaked curve. The sex difference is at any rate in the same direction in both sets of data. The ages at which peak height velocity was reached averaged, in our data, 14.1 \pm 0.13 years for boys and $12 \cdot 1 \pm 0.14$ years for girls, and in Deming's, $13 \cdot 5 \pm 0 \cdot 18$ for boys and $11 \cdot 5 \pm 0.20$ for girls. The sex difference in age of peaking is thus $2 \cdot 0$ years in our data and exactly the same in Deming's. Deming's children appear to reach their peak about six months earlier than ours. This is almost certainly a true finding and not due to any difference in fitting the curves, for the average menarche of the Denver girls was $12 \cdot 6$ years and of our Harpenden Growth Study girls 13.2 years. The difference is probably attributable to socioeconomic and nutritional factors. In both sexes and in both sets of data the standard deviation of the age of peak velocity was 0.9 year, which closely approximates the usual figure found for the standard deviation of age at menarche.

There is a tendency for the earlier maturing boys to have a greater peak height velocity than the later maturers, as can be seen from Fig. 5B. The same is true of girls. The regression of peak height velocity on the age at

TABLE I

Peak Height Velocities ('Instantaneous') and Age at Peak in 49 Boys and 41 Girls of the Harpenden Growth Study

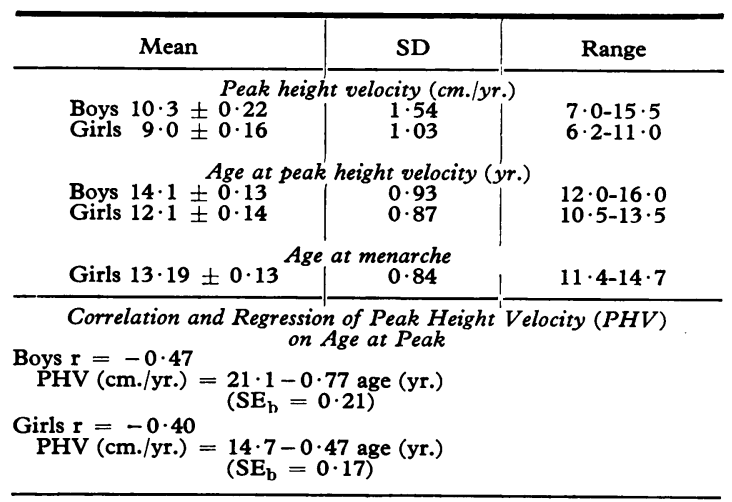

Correlation and Regression of Peak Height Velocity (PHV) $\mathbf{r}=-0.39$ and Age at Menarche

$\mathrm{PHV}(\mathrm{cm} . / \mathrm{yr})=.15.5-0.50$ age $(\mathrm{yr}$.

which it occurs is plotted in Fig. 6 (A, boys; B, girls). The regression equations and the correlations are given in Table $I$. This relationship has been universally found in studies of adolescence, from Boas (1932) and Shuttleworth (1939) to Simmons and Greulich (1943) and Deming (1957). Our regressions and Deming's are not significantly different. A mean drop in peak height velocity of $0.77 \pm 0.21 \mathrm{~cm}$. $/ \mathrm{yr}$. for each year of age occurs in our boys and a drop of $0.59 \mathrm{~cm}$. $/ \mathrm{yr}$. for each year of age in Deming's boys. Our girls drop by $0.47 \pm$ $0.17 \mathrm{~cm} . / \mathrm{yr}$. on average, and Deming's by $0.93 \mathrm{~cm}$. $/ \mathrm{yr}$.

The breadths of the curves for early and late-maturers (the dimension that would correspond to the standard 
(A)
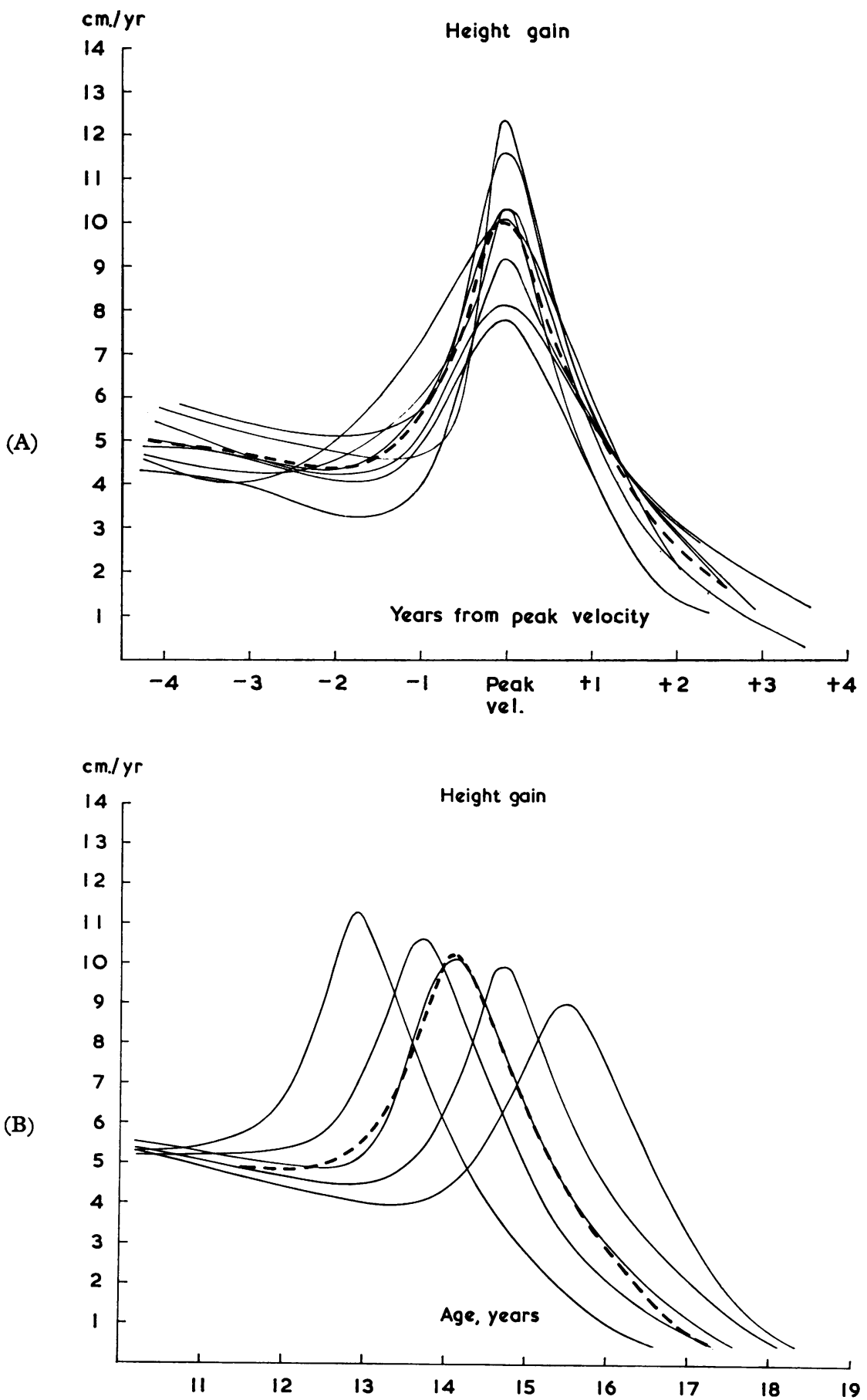

FIG. 5.-(A) the group of height velocity curves of Fig. $4 A$ arranged so that the peaks are vertically above each other with the average curve for this group drawn as a dashed line. (B) the average curves of the five groups of boys, with the over-all average curve (dashed) resulting from their combination. 


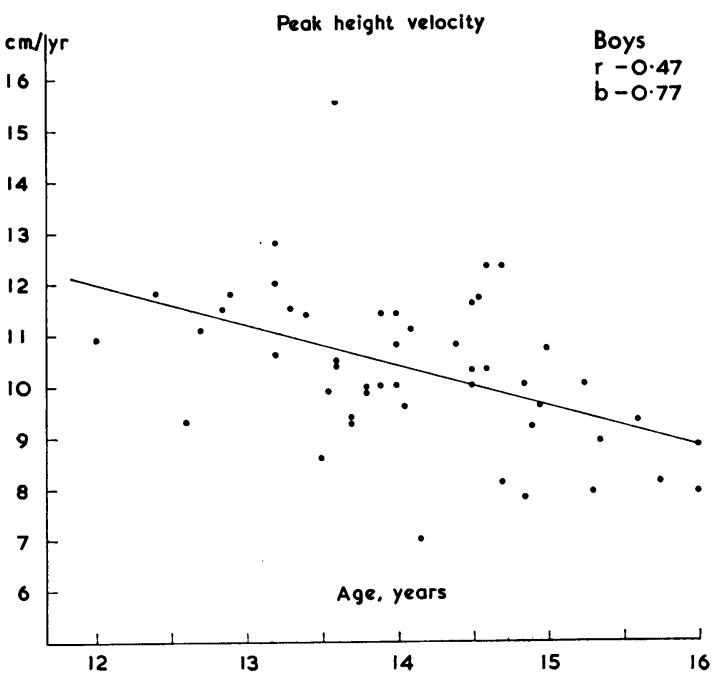

(A)

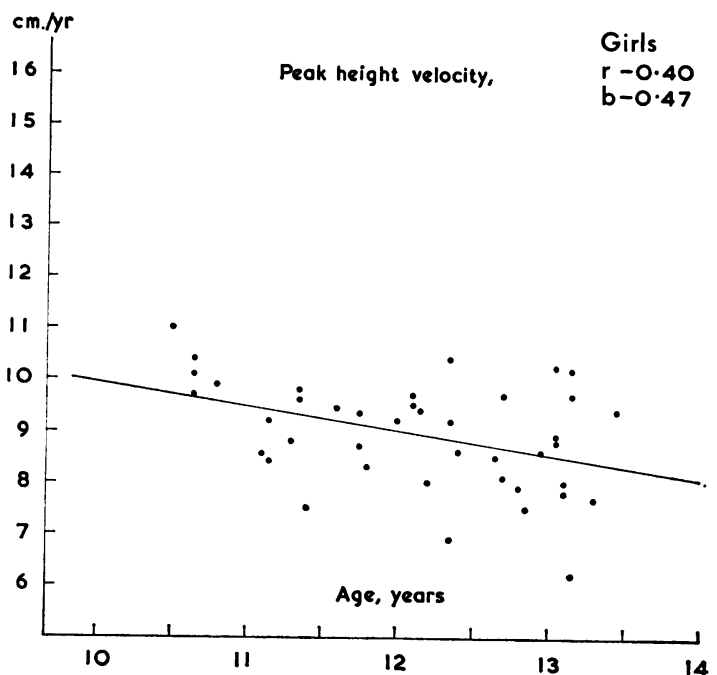

(B)

FIG. 6.-Regression of peak height velocity on age at which peak occurs. (A) boys; (B) girls.

deviation in a Gaussian distribution curve) are very similar, as can be seen in Fig. 5B. There seems to be no consistent tendency for the earlier maturers' curves to be thin and peaked and the late maturers' to be flat and round, or vice versa. Height and breadth of the individual curves in general seem to be independent. Thus no problem arises from this aspect in combining all the curves to make our standard.

One further problem does arise, however. In some of the height velocity curves of the boys there seemed to be a dip downwards just before the adolescent acceleration began. This occurred chiefly in the late-maturing boys. The velocity is decreasing at this time anyway, and it is exceedingly difficult to know whether what looks like an added rate of decrease-a greater deceleration-is real or simply an accumulation of slight errors and a coincident seasonal effect. Taking the sceptical view we have smoothed any such dip out of our standards; but we would like to go on record that this is a subject that needs investigation. If there is a real dip, it might be merely due to differential acceleration of trunk and legs (see Tanner, 1962a, p. 12), or, more interestingly, to some alteration in endocrine balance.

Various authors (e.g. Grant, 1964) have thought such a dip may occur, and Bayley (1956) actually put it into her height velocity standard for boys, though not for girls. Her standards are particularly interesting because they were the first to present a 50th centile velocity curve over the whole age range and to endeavour to differentiate early, average, and late-maturing children. Their use on clinical material has been extensively illustrated in Bayer and Bayley (1959). The velocity curves are based on about 300 healthy Californian children followed from birth to maturity (the Tuddenham and Snyder (1954) data plus two other Berkeley studies).
Weight. Individual velocity curves of weight at adolescence were obtained by the same smoothing procedure as for height. The individual curves were combined to obtain the shape of the average curve. Average figures for peak weight velocity and age at peak weight velocity were calculated from the individual curves.

Smoothing the weights presented much greater uncertainties than smoothing the heights. Seasonal variations in velocity were more marked, weight loss occurred from time to time, and in many cases no clear final asymptote was present. The difficulties were particularly marked in girls, whose fat content varied with whim and weather.

A number of individual curves are given in Fig. 7A, and the composite curves for five groups, together with the final over-all average curve, in Fig. 7B. The composite curves are surprisingly regular, but clearly they must be only approximate. In the clinic we find body weight the least helpful of all body measurements after the first two years of life. Fat-free weight would

TABLE II

Peak Weight Velocity ('Instantaneous') and Age at Peak in 46 Boys and 33 Girls of the Harpenden Growth Study

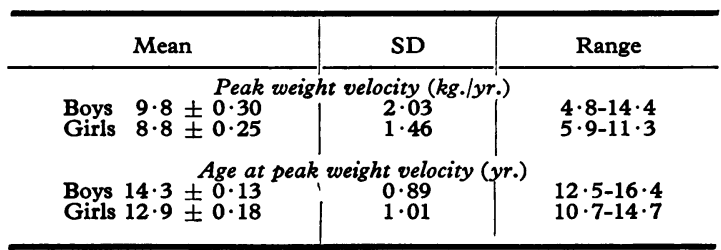



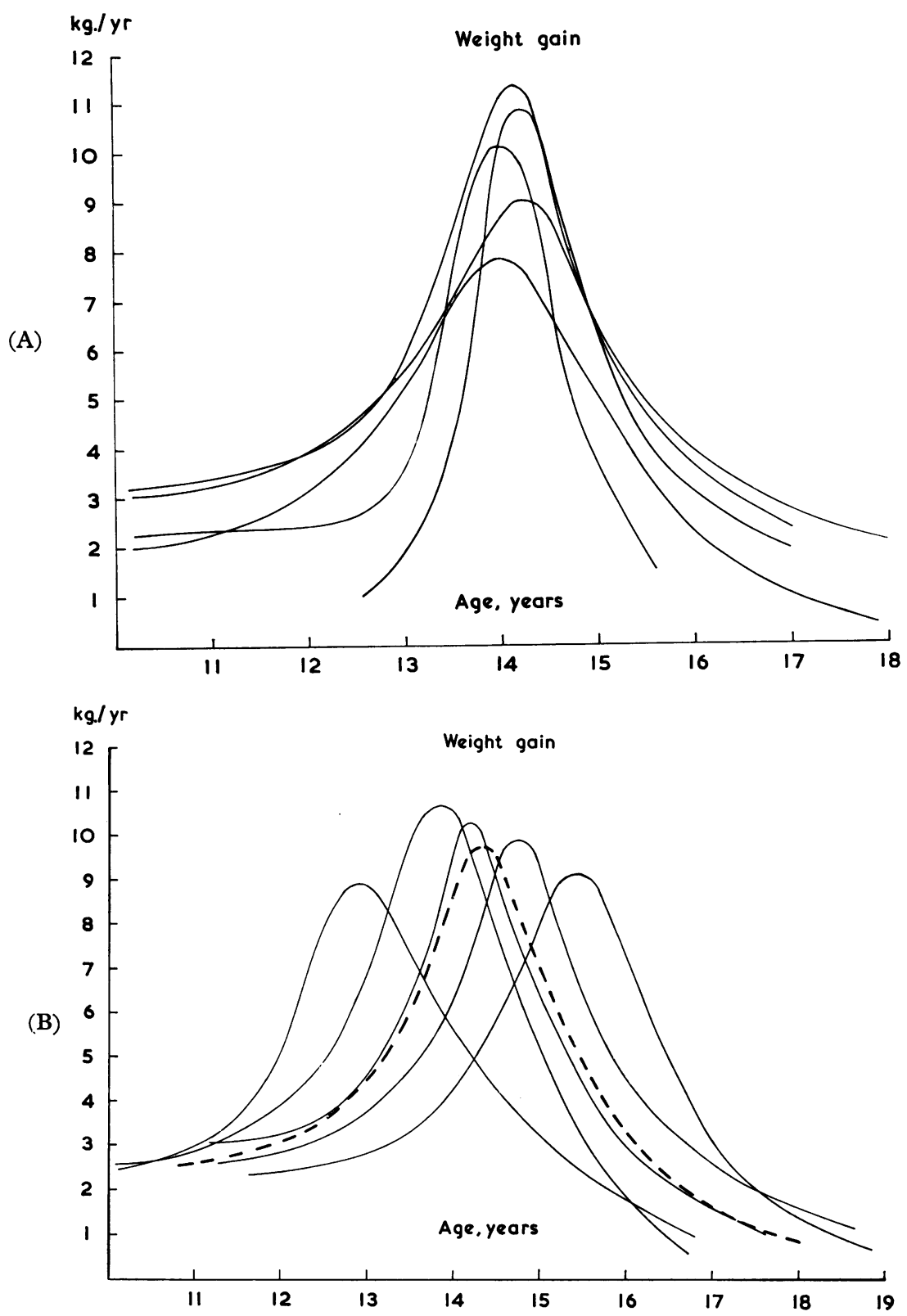

FIG. 7.-(A) weight velocity curves of 5 average-maturing boys. (B) average curves of five groups of boys, with overall average drawn as dashed line-compare Fig. 5B.

undoubtedly be more useful, but we do not at present have measuring techniques that are simple enough for routine clinical use.

The peak weight velocity averaged $9 \cdot 8 \pm 0 \cdot 30 \mathrm{~kg}$. $/ \mathrm{yr}$. in boys and $8 \cdot 8 \pm 0.25 \mathrm{~kg}$./yr. in girls (Table II). Bayley's figures, which for methodological reasons would be expected to be slightly lower than ours, are approximately $8 \mathrm{~kg}$. $/ \mathrm{yr}$. for boys and $6 \mathrm{~kg}$. $/ \mathrm{yr}$. for girls. Peak weight velocity occurs at a slightly older age than peak height velocity, and because of this our sample of boys and girls is not quite the same for height and weight. Of the 49 boys, 3 had not completed their weight spurt though 
their height spurt was over: these have been omitted in the weight velocity. A rather larger number of the 41 girls had failed to complete their weight spurt, and so of the original 41 only 31 appear in the weight curves, and an extra 2 have been added who were not in the height curves. The average age at peak weight velocity was $14.3 \pm 0.13 \mathrm{yr}$. in the boys and $12.9 \pm 0.18 \mathrm{yr}$. in the girls. The 46 boys present both for height and weight peaked in weight $0.25 \mathrm{yr}$. later than in height; the 31 girls peaked $0.63 \mathrm{yr}$. later.

As expected, there is a close relation between the age of peak weight velocity and the age of peak height velocity. The correlation ooefficients are 0.93 for the 46 boys and 0.82 for the 31 girls. Considering the uncertainty involved in fitting the curve, these correlations are high, the boys' quite remarkably so. Indeed, this is indirect evidence that our fits were quite accurate, for such a result could not have happened if much error were involved in determining either peak. The lower correlation in girls may be due purely to sampling error; but more likely it reflects difficulties in fitting the weight curve, and also a greater contribution of fat increase to the peak, resulting in peaks that are more heterogeneous in terms of tissue. There was a much lower correlation between the size of the height velocity and weight velocity peaks, than between the ages at which they occurred. The actual peak velocities in height and weight correlated only 0.29 in the boys and 0.18 in the girls.

The relation between peak volocity and age of peaking, seen so clearly in height, is not present in weight. The correlations are entirely insignificant, being -0.04 in boys and +0.02 in girls. Neither is there any relation between peak weight velocity and age at menarche. Part of this lack of correlation may be because weight represents such a conglomeration of differently growing tissues. Actually the 2nd, 3rd, 4th, and 5th groups of boys, in terms of age-at-peaking, did show the same relations as for height, the peaks descending in the order of the groups; but the earliest-peaking boys had a very low peak. Amongst the girls even this degree of regularity did not occur. The sex difference in pattern may come from the weight peak being more governed by fat in girls, and by muscle (which is more linked to stature) in boys.

Some of the individual weight velocity curves seemed to show a plateau immediately before adolescence, corresponding to the dip at this time seen in some of the height velocity curves. We were somewhat tempted to make the final 50th centile weight velocity curves less simple in shape than they appear in Fig. 9 (below). Instead of the gradual rise from 5 till adolescence, there may perhaps be a slightly steeper rise from 5 till about 8 in the girls or 10 in the boys, followed by a plateau till the adolescent spurt begins. A number of authors have supposed that this occurs, but they nearly all were operating with cross-sectional data. It seems that a lot depends upon the fat situation; where children become fat the weight may indeed rise in a two-phase manner, the first phase ( 5 to $8 / 10$ ) being chiefly due to fat increase, and the second to the adolescent spurt. However, we judged this effect to be neither sufficiently clear nor sufficiently general to be incorporated into our standards, so Fig. 9 ignores it.

\section{Individual-type Centiles for Height and Weight Attained}

The Harpenden Growth Study data were used to determine the shape of the individual height and weight curves at adolescence, but they are not suitable as figures on which to base standards. For one thing, they are obviously too limited in number, and for another they come from boys and girls living in a children's home. The home is an extraordinarily good one, in which the children live in 'family units' consisting of a dozen boys and girls of all ages supervised by a housemother and her younger assistant. Most of the children are from broken homes, usually manual working class. Most enter at between 3 and 5 years and stay in the home till between 15 and 18 . The home is well situated in extensive grounds, the food is excellent, and the children attend the schools in the town in the ordinary way. We think it very unlikely that the shapes of these children's adolescent growth curves are in any way unusual, but menarche does occur slightly later than in the London County Council children. For this reason in making the standards we have moved the age of peak velocity of height and weight earlier by $0.2 \mathrm{yr}$. in both sexes. This means that the average age of menarche in the standards is 13.0 years, and peak height velocity 11.9 years in girls and 13.9 years in boys. These figures are probably closely representative of urban children of average economic status in Southern England in the early 1960's.

When considering individual-type standards we have stressed the velocity rather than the heightattained curves, because the most convenient, if apparently roundabout, way of constructing the individual-type height-attained curves is by first finding the equivalents in velocity terms, and then simply integrating them. For drawing the typical individual velocity curves then, the figures for the Harpenden Growth Study smoothed individual curves were used from 10 onwards in boys and 8 onwards in girls but with the peak velocities placed 0.2 years earlier. Below age 10 in boys and 8 in girls the median velocities were those of the Child Study Centre children. The two sets of data linked up well, and little smoothing was necessary. The curves are shown in Fig. 8 and 9 and the data are given in Appendix Tables I and II.

The paper by Deming and Washburn (1963), in which they fitted the Jenss curve to longitudinal records of supine length in 40 boys and 40 girls from 


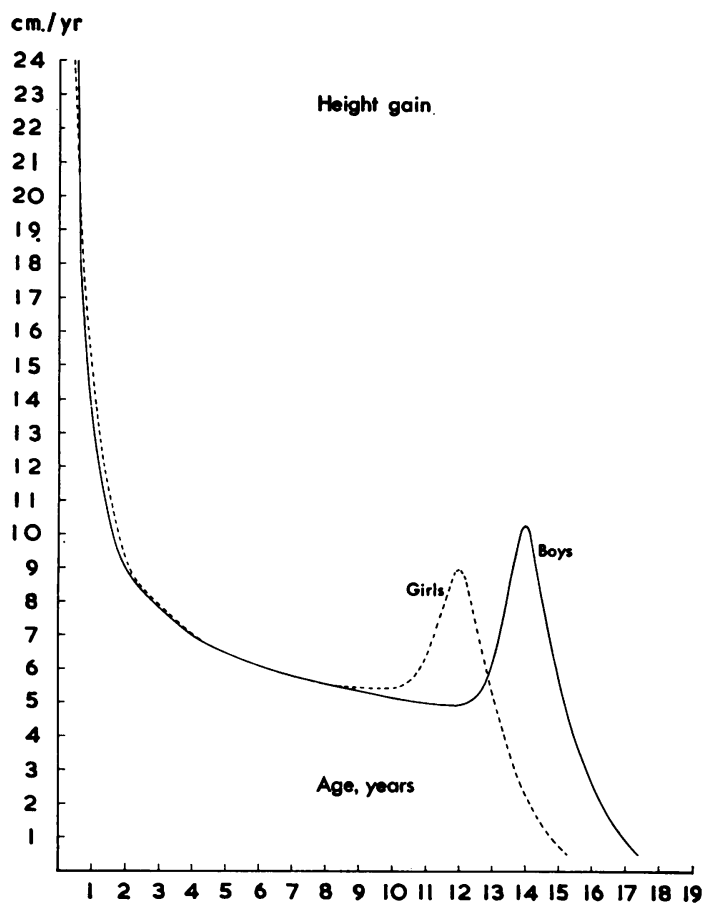

FIG. 8.-Typical individual velocity curves for supine length or height in boys and girls. These curves represent the velocity of the typical boy and girl at any given instant. For construction see text. birth to 8 years, was very helpful in defining the height velocities during the first few years after birth. Deming shows the boys' velocity as greater than the girls' at birth, but becoming equal at about 7 months and subsequently less until about 4 years. This agrees with our data, and with the majority of the published data. The sex difference is best thought of, perhaps, in terms of acceleration, boys decelerating harder than girls during the first 4 years. The published reports are not consistent as to which sex has the greater velocity from 4 till adolescence, so we have made them identical, pending further information.

In weight the pattern is very similar. The boys' velocity is greater at birth, but becomes equal to the girls' at about 8 months and then gradually drops below (Deming and Washburn, 1963; Ministry of Health, 1959). The majority of the data seem to indicate that the boys' weight velocity then continues to stay a little below the girls' right up to adolescence. Weight velocity depends on exogenous factors more than height velocity, however, so that we cannot assume that this sex difference applies to all populations or under all conditions.

The curves in Fig. 8 and 9 represent the instantaneous velocity, at any given moment of age, of the typical boy and girl. The individual-type distance standard we are searching for is the integral of this instantaneous velocity curve.

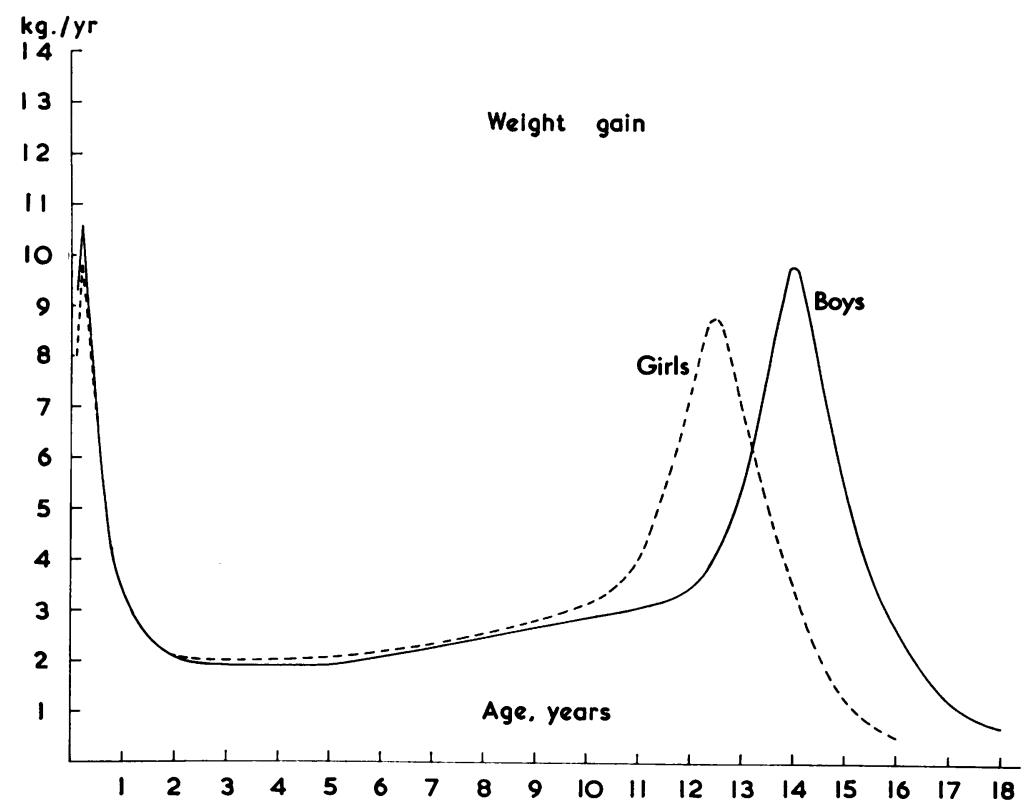

FIG. 9.-Typical individual velocity curves for weight in boys and girls. These curves represent the velocity of the typical boy and girl at any given instant. For construction see text. 
The accumulated height at each year of age was, therefore, calculated by taking the mean length at 1 month and adding on successively the increments from the velocity curves of Fig. 8, less the centimetre difference in changing from supine length to standing height. The resulting 'typical-individual' distance curves had to be subjected to a further smoothing, carried out to fit the shape of the integrated curve derived from the small-scale longitudinal data onto the pre- and post-adolescent norms of height and weight derived from the large-scale cross-sectional data. The integrated velocity curves were plotted on the original cross-sectional curves for height and weight attained, after the manner illustrated in Fig. 2 (right) on page 459. At adolescence the curves were naturally very different, but before and after adolescence they corresponded closely and were made to correspond exactly by repeated cycles of smoothing first one and then the other. A similar process was followed for the weight curve.

The 'typical-individual' curves thus resulting for girls' and boys' height and weight attained are shown in Fig. 10 and 11. The typical girl is slightly shorter than the typical boy at all ages till adolescence; she becomes taller shortly after age 11 and remains so till age 14. She is lighter at birth but begins to be heavier at about age 8 and remains so till about $14 \cdot 5$ years.

The boy who has travelled up the 50th centile before adolescence, who has his adolescent spurt at exactly the average age, and who ends up as a 50th centile adult follows the typical individual curve of Fig. 10. But we wish also to provide lines in our standards for the boy who has travelled up the 90th centile, or the 10th. This we can do, provided always that we are thinking of a 90th centile boy who has his spurt at the average age and ends up at the 90th centile. The shaded bands shown in Fig. 12 and 13 characterize such children, using the usual centiles. Thus a small 3rd centile child will follow the edge of the lowest shaded band throughout adolescence if he has (a) an adolescent spurt of average velocity and (b) if he has it at the average age. If he spurts early, he will still have a curve of approximately the shape of the shaded channels, but with the time-base shifted. Degree of advancement can, in fact, be judged in this way. The early-spurting child's curve is not precisely parallel to the curve traced out by his appropriate centile at the average age, because the peak velocity of the spurt is greater for early than for late maturers, irrespective of their centile position. Thus the early-spurting child will rise slightly more steeply than his shaded centile channel, and the late-maturing slightly less steeply. (Our charts are the converse of Bayer and Bayley's

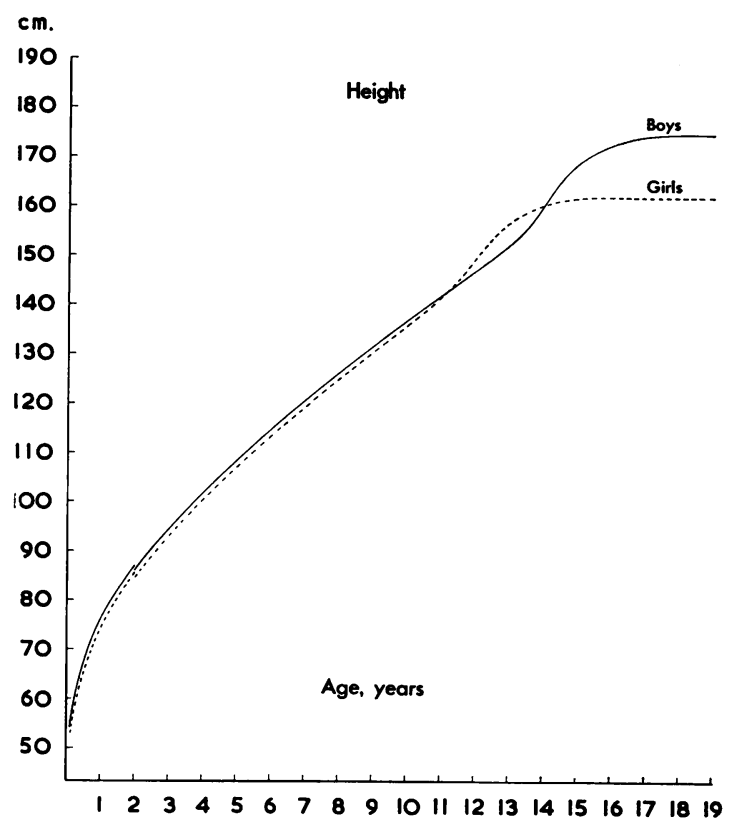

FIG. 10.-Typical individual height-attained curves for boys and girls. (Supine length to the age of 2.) Integrated curves of Fig. 8.

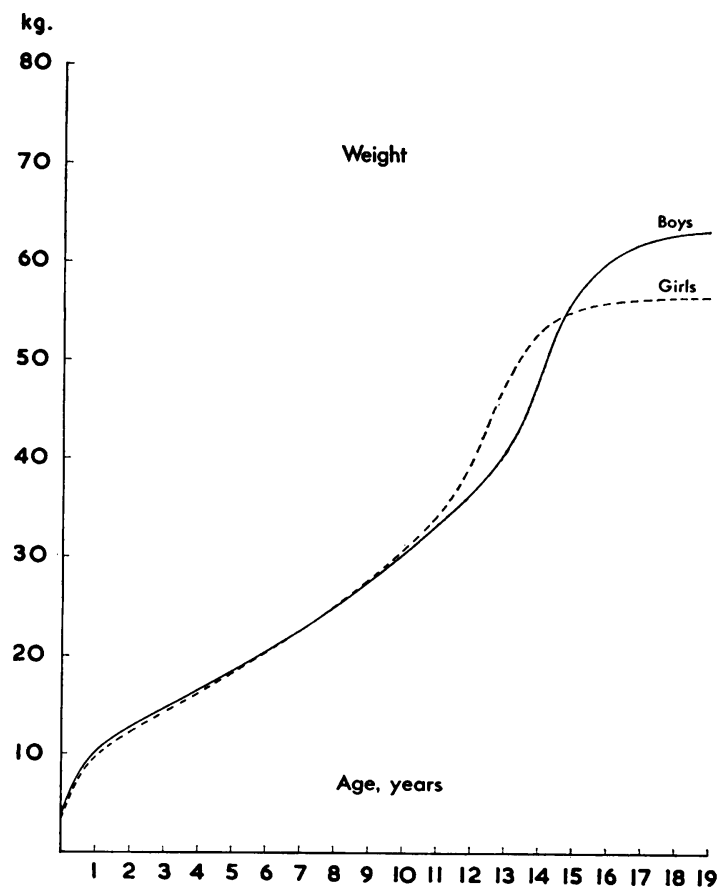

Fig. 11.-Typical individual weight-attained curves for boys and girls. Integrated curves of Fig. 9. 


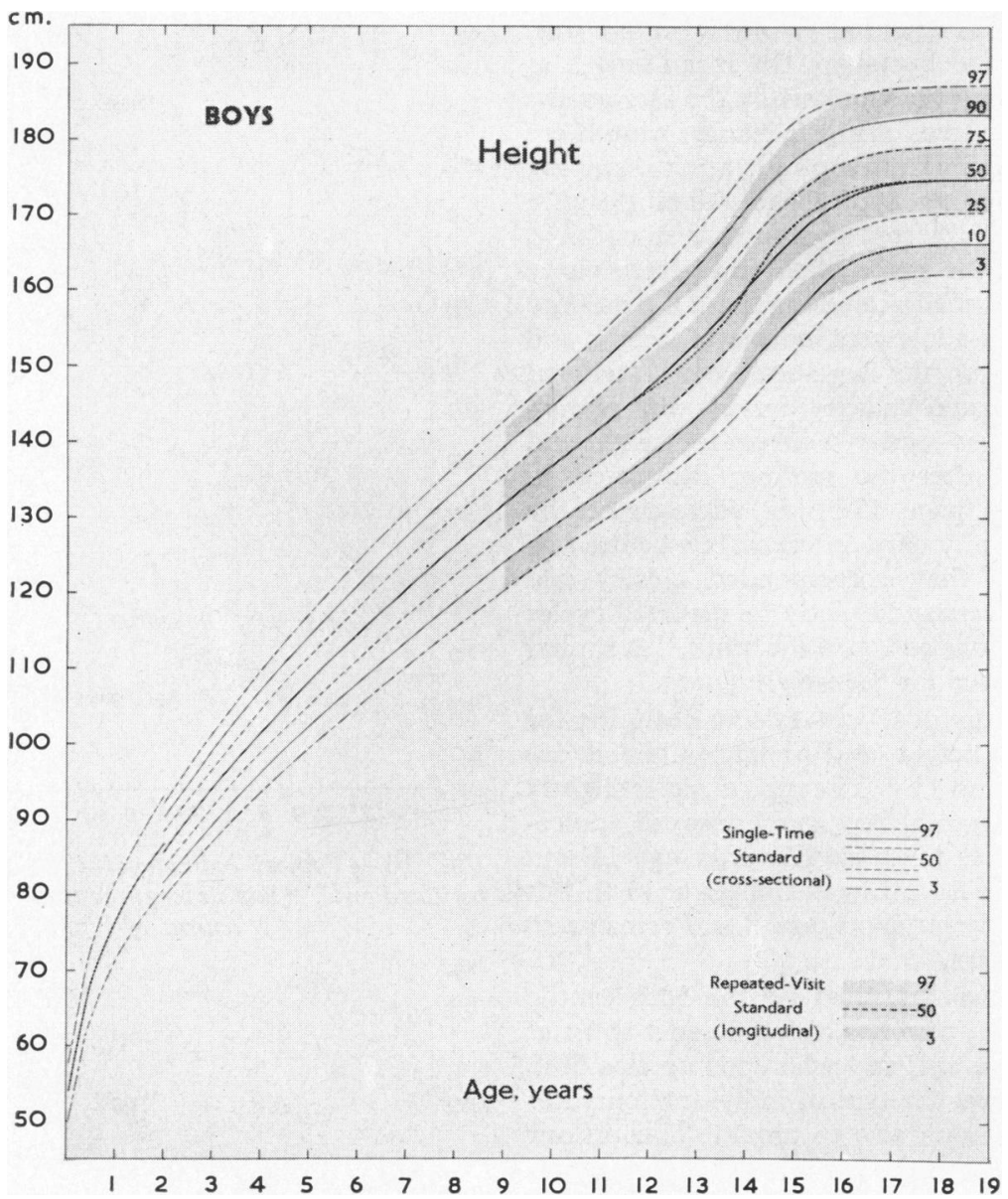

Fig. 12.-Cross-sectional and individual-type (shaded) standards for height attained at each age (boys). For construction see text.

(1959), where growth lines are presented for the 50th centile child who is early-maturing, the 50th who is average-maturing, and the 50th who is late-maturing.)

We can regard these individual-type curves in another light. The reason the conventional centiles fail to represent the growth of an individual during adolescence is the phase-difference effect discussed above. The individual-type lines therefore represent the centiles we would obtain if we were able to eliminate the phase-difference effect, and this we can do by plotting the height attained against a measure of biological maturity instead of against chronological age. The typical individual curve is in fact the 50th centile curve in a conventional distance standard in which at adolescence height attained is plotted against developmental age, as measured in years before or after peak height velocity (PHV). Using this same age base we can calculate centiles in the usual way and this is in fact how we arrived at the shaded lines in Fig. 12 and 13. For height we used the smoothed SD of height at each year of age before or after PHV, that is $\mathrm{PHV}-3 \mathrm{yr}$., $\mathrm{PHV}-2 \mathrm{yr}$. PHV -1 yr., and so on. Naturally this is only necessary at adolescence; before and after it the individual-type curves coincide with the conventional ones.

Our standards for height, shown in Fig. 12 and 13, are tabulated in Appendix Tables III and V. The regular centile lines represent the conventional or cross-sectional standards, and the shaded bands, together with the dashed line going up the middle of 


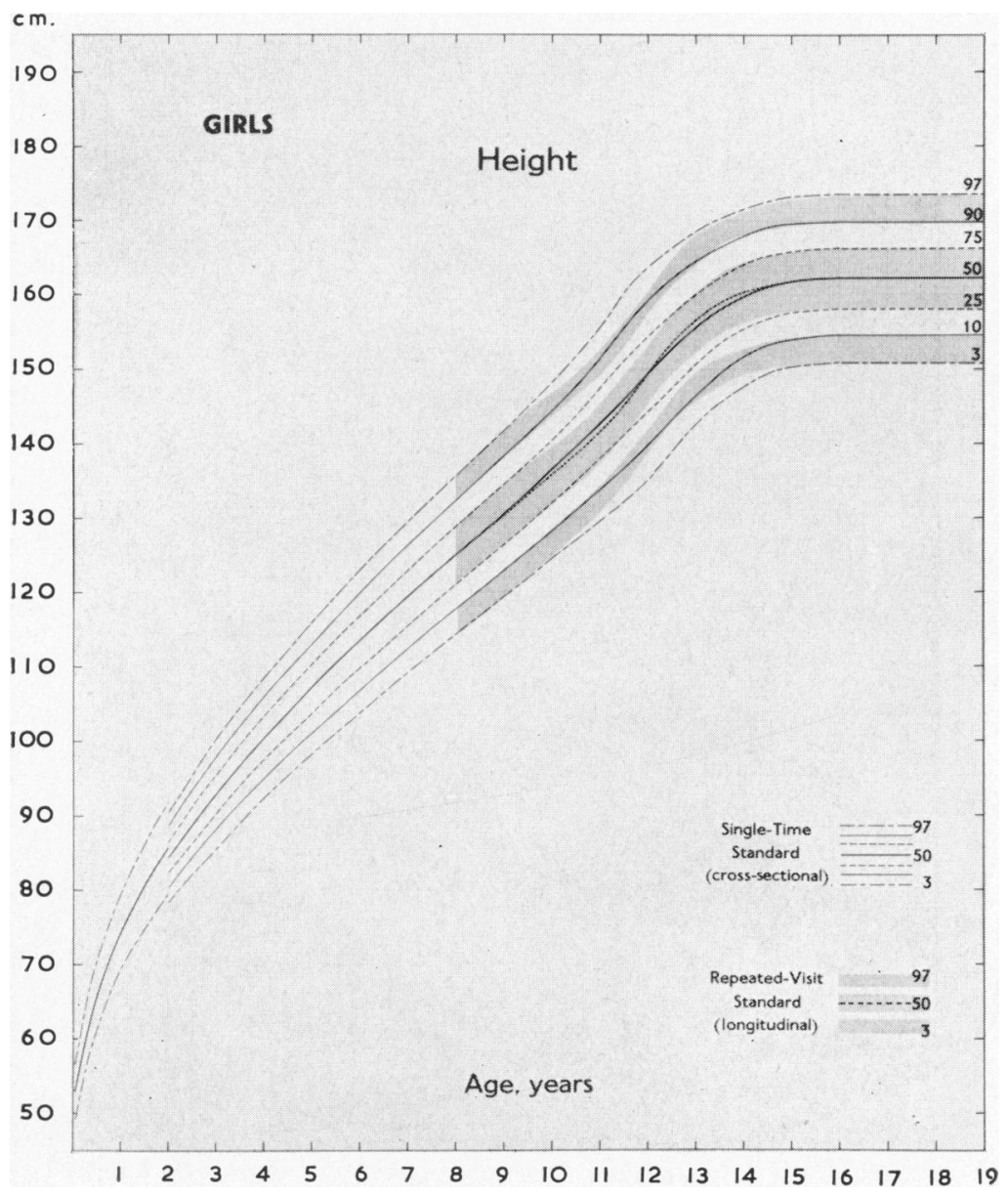

Fig. 13.-Cross-sectional and individual-type (shaded) standards for height attained at each age (girls). For construction see text.

the central band, represent the individual-type standards. The cross-sectional centiles should be used when a child is seen once only and when no estimate of developmental age is available. But when a child is seen on several occasions, then the course of his growth at adolescence, when plotted either on a chronological or a developmental age base, is best compared with the individual-type shaded areas, for it is up these steeper channels that an individual actually passes. When a child is seen once only, but with an estimate of developmental age available, the situation lies somewhere between the cross-sectional and the individual interpretations. If one had a peak-height-velocity-age available, then of course, one would simply use the shaded bands. But on a single occasion this can never be; the usual measure available is skeletal age. Skeletal age is closely, but not perfectly, related to peak-heightvelocity-age, so that the centile channels for skeletal age lie in an intermediate position between the two systems we have illustrated. The same is true of menarcheal age (years before or after menarche) or secondary sex character age. The best advice for the single occasion seems to be to plot both against chronological age (interpret on line centiles) and skeletal age (interpret on shaded channels, but conservatively) and use both results in making the final assessment as to whether or not the child is probably normal.

The standards for weight attained are given in Fig. 14 and 15 and tabulated in Appendix Tables IV and VI. The conventional weight-attained centiles 


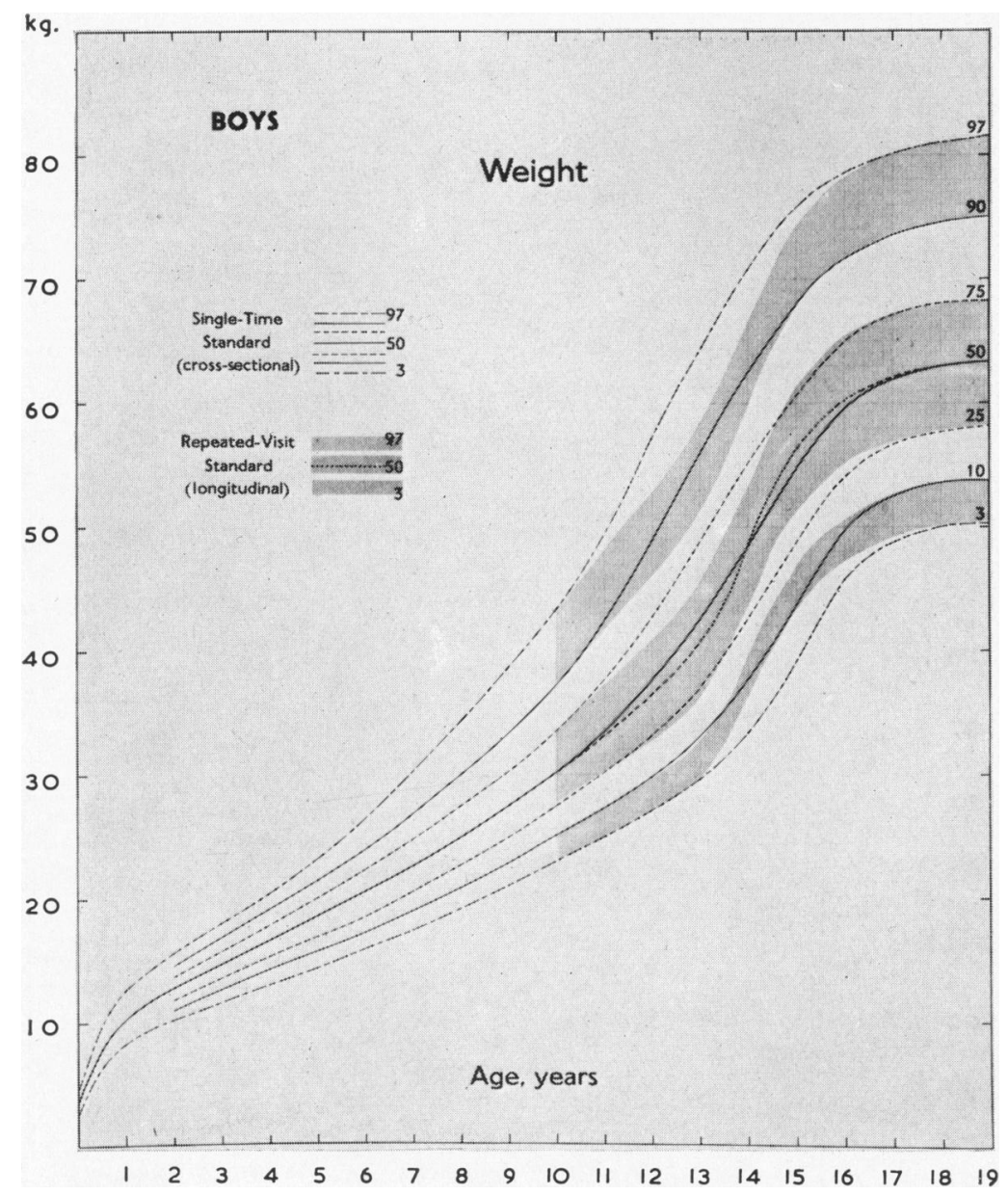

Fig. 14.-Cross-sectional and individual-type standards for weight attained at each age (boys). For construction see text.

were calculated directly from the raw data, since the distribution of weight is skewed, and when this is so, the use of the SD is erroneous. To produce the individual-type channels over adolescence the same technique was used as for height. This is an approximate operation only, but centiles of weight at this age are in any case only of dubious value.

One remark must be made about the relation of our cross-sectional and individual-type values. The 50th centile curves of the two had clearly to be symmetrical in the sense of enclosing equal areas before and after they crossed. We first constructed the individual-type 50th centile in the way described above, and then found that when the cross-sectional standards were superimposed upon it this criterion of symmetry was not quite met. Symmetry could most simply be obtained by adjusting the cross-sectional standards to slightly higher values at adolescence. This is equivalent to making the adolescent spurt occur some two months earlier than the 1959 figures demanded. In view of the known secular trend, we have accepted this change, which is almost identical to the change that actually occurred in London standards between 1954 and 1959. Thus our figures may be taken to refer to London children's growth as estimated for 1965.

In many European countries children growing up in villages in the countryside are smaller than those in the big towns, but in England such a difference is very slight, if present at all. Children in the North of England may be a trifle smaller than those in the South, and for the Scottish lowlands and Northern Ireland the difference may be a little greater. These 


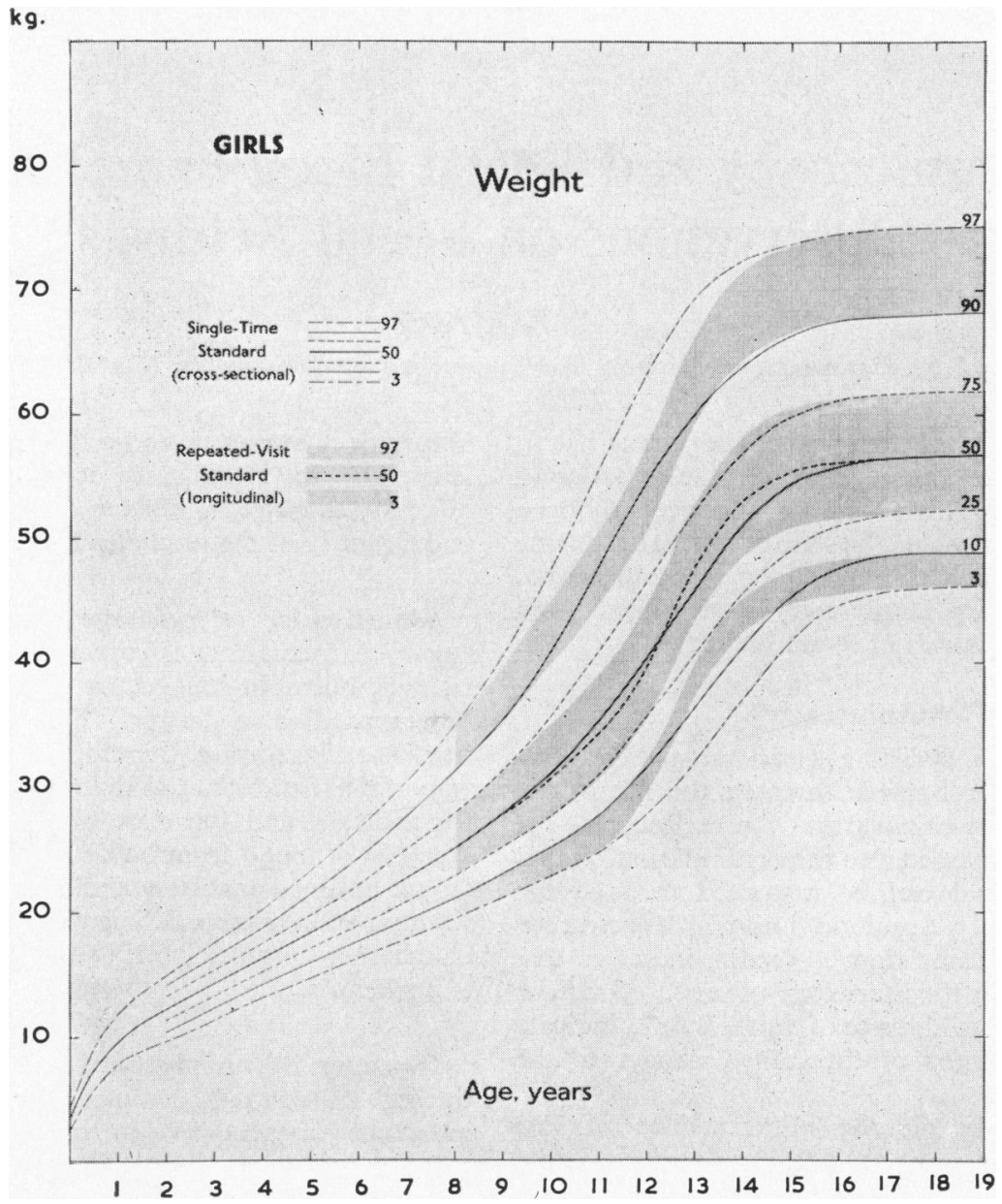

FIG. 15.-Cross-sectional and individual-type standards for weight attained at each age (girls). For construction see text.

standards can be regarded as quite suitable for all mind, probably for Scottish and Irish children English children, and, with these precautions in also. 